\title{
Applications of information and communications technologies to public health: A scoping review using the MeSH term "public health informatics"
}

\author{
Arjun Kumar Bhattarai ${ }^{1}$, Aein Zarrin $^{1}$, Joon Le $^{1}$ \\ 1. Health Data Science Lab, School of Public Health and Health Systems, University of Waterloo, \\ Waterloo, Ontario, Canada \\ *Joon Lee, Health Data Science Lab, School of Public Health and Health Systems, University of Waterloo, Waterloo, \\ Ontario, Canada. joon.lee@uwaterloo.ca
}

\section{Abstract}

Objective: To investigate the public health domains, key informatics concepts, and information and communications technologies (ICTs) applied in articles that are tagged with the MeSH term "public health informatics" and primarily focus on applying ICTs to public health.

Materials and Methods: The MeSH term "public health informatics" was searched on MEDLINE-PubMed. The results of the search were then screened in two steps in order to only include articles about applying ICTs to public health problems. First, articles were screened based on their titles and abstracts. Second, a full-text review was conducted to ensure the relevance of the included articles. All articles were charted based on public health domain, information technology, article type, and informatics concept.

Results: 515 articles were included. Communicable disease monitoring ( $\mathrm{N}=235)$, public health policy and research $(\mathrm{N}=201)$, and public health awareness $(\mathrm{N}=85)$ constituted the majority of the articles. Inconsistent results were found regarding the validity of syndromic surveillance and the effectiveness of $\mathrm{PHI}$ integration within the healthcare systems.

Discussion: PHI articles with an ICT focus cover a wide range of themes. Collectively, the included articles emphasized the need for further research in interoperability, data quality, appropriate data sources, accessible health information, and communication. The limitations of the study include:1) only one database was searched; 2 ) by using MeSH tags as a selection criterion, $\mathrm{PHI}$ articles without the "public health informatics" MeSH term were excluded.

Conclusion: Due to the multi-disciplinary nature of $\mathrm{PHI}, \mathrm{MeSH}$ identifiers were not assigned consistently. Current MeSH-tagged articles indicate that a comprehensive approach is required to integrate $\mathrm{PHI}$ into the healthcare system.

Keywords: Public health informatics, population surveillance, health policy, environmental monitoring, public health

Abbreviations: Public Health Informatics (PHI), Public Health (PH) 
Correspondence: Joon Lee, Health Data Science Lab, School of Public Health and Health Systems, University of Waterloo, Waterloo, Ontario, Canada. joon.lee@uwaterloo.ca

DOI: 10.5210/ojphi.v9i2.7985

Copyright @2017 the author(s)

This is an Open Access article. Authors own copyright of their articles appearing in the Online Journal of Public Health Informatics. Readers may copy articles without permission of the copyright owner(s), as long as the author and OJPHI are acknowledged in the copy and the copy is used for educational, not-for-profit purposes

Public Health Informatics (PHI) is a discipline of health informatics that integrates information and communications technology (ICT) and public health (PH) [1]. The field of informatics pursues collection, storage, retrieval, and analysis of data [2]; this type of manipulation of data enables informaticians to derive meaningful information regarding certain aspects of the data sources, such as trends and predictive models [2]. Moreover, informatics allows users to access informative content more effectively and efficiently [2]. One important corollary of the definition of informatics above is that informatics may not necessarily involve any use of ICT, although modern informatics tends to emphasize the role of ICT.

$\mathrm{PH}$ is defined as the organized efforts of society to keep people healthy and prevent injury [3]. $\mathrm{PH}$ involves a combination of programs, services, and policies that promote the health of the population [3]. To improve the efficiency and effectiveness of public health practice, as well as to make information-driven decisions, public health practitioners and researchers must be able to gather and analyze information in a timely yet reliable manner; moreover public health research and policy-making relies on tools that utilize such information to disseminate new knowledge [4]. Aiming to accommodate both informatics and public health, PHI could be a pathway to enhance public health practice. In other words, PHI could be considered as a potential tool to facilitate public health goals, such as effective health monitoring and surveillance, enhanced decisionmaking, and improved population health. (2 \& 5)

Although the term PHI was first used in literature in 1995 [2], research studies focusing on PHI proliferated in early 2000s; this may be attributed to several events in the 21st century, such as anthrax bio-terrorism in 2001 [1], alongside the rapid advent and innovation of modern ICTs [1]. This increase in the number and effectiveness of PHI studies resulted in the creation of MeSH term "public health informatics" in 2003 [6]. MeSH term is a vocabulary used to facilitate the consistency of health research categorization and aims to enhance the availability of existing literature [6]. Followed by a complete review of the titles, contents, and references of research studies, MeSH terms are assigned to the group of articles that predominantly focus on the field or topic represented by the corresponding MeSH identifier [6]. Therefore, it is logical to expect that most PHI research articles would be tagged with the MeSH term "public health informatics". However, it cannot be assumed that all PHI articles would be tagged with the MeSH term "public health informatics", particularly any PHI studies published prior to the introduction of the MeSH term in 2003, not to mention that there could be a delay with MeSH term tagging in practice. 
Dixon et al., have conducted a scoping review limited on the recent trends in global health and PHI between 2012 and 2014; however, reviews that scope the field since the creation of its MeSH term do not currently exist [7]. Additionally, the present review is aiming to specifically focus on PHI whereas Dixon et al. primarily examined global health informatics.

Considering the criteria for MeSH term assignment and the proliferation of PHI in the past two decades, a scoping review of the articles tagged with the MeSH term "public health informatics" allows an overview of the studies focused on PHI. This overview will be used to map the literature to identify trends, potential areas of research, and knowledge gaps in MeSH-tagged PHI articles. In order to do so, sub-categories and different domains of PHI should be investigated. Thus, the objective of the present review study was to investigate the public health domains, key informatics concepts, and information technologies applied in articles tagged with the MeSH term "public health informatics". Furthermore, we focused only on PHI articles with a strong ICT angle, to be in line with the direction of modern PHI.

\section{Methods}

The MeSH term "public health informatics" was utilized for the initial search. As MeSH terms are a unique feature of MEDLINE, only this database was used for this review. This literature search was followed by a two-step screening process (described below) to apply inclusion and exclusion criteria. The inclusion criteria were any article that applies, investigates, analyzes, describes, or assesses ICT within the field of public health and was originally published in English. As a result, articles that did not utilize or discuss a direct application of ICTs to solve $\mathrm{PH}$ issues were excluded from the study. This exclusion criterion removed studies that only applied basic statistics to a health dataset, for example. Any article that mainly dealt with other branches of health informatics such as biomedical, clinical, nursing, or consumer health informatics or any article published originally in a non-English language was excluded.

Initially, two researchers reviewed the title and abstract of each of the 1072 articles independently and labeled them as either "Include" or "Exclude". Studies that did not seem to fit either of the categories were labeled as "maybe". Moreover, if the title and abstract did not provide sufficient information on the content of the literature, the full text of the article was skimmed. Note that those studies that were labeled as "exclude" or "include" by both researchers were excluded or included, respectively, prior to the second round of screening. In the second round of screening, the two reviewers met in person to reach consensus regarding the articles they disagreed on; most of which had multiple focuses in addition to PHI. Only the ones that primarily examined ICT in public health were included, and the purpose of the deliberation between the reviewers was to decide whether this was indeed the case. PHI was distinguished from other specialties of informatics, particularly bio-medical informatics, by its focus on wide range of preventive interventions within populations and government-based operative context [1]. Following the meeting, the full-text of each included article was studied to ensure their eligibility for the present study.

The second step of the literature search was "charting" the data; this method was used by Ritchie and Spencer to scope the gaps or common themes in certain bodies of knowledge in health research [8]. The full texts of the research articles that were labeled as "include" by both reviewers or discussed to be included in later meetings, were studied, analyzed, and summarized 
in a chart in terms of their citation data, year of publication, article type, public health domain, ICT, and informatics concept. Public health domains consisted of 8 categories that would satisfy all the goals of public health [3]: communicable diseases monitoring, non-communicable diseases monitoring, emergency response, injury surveillance, natural disaster management, environmental health, public health awareness, and public health policy and research. The ICT categories summarized common information technologies used/discussed in each article. The 10 ICT categories include: detection/prediction (DP) algorithm, electronic registry, e-mail, geographic information system (GIS), mass media, mobile phone, natural language processing (NLP), analytical software, landline telephone, and websites. The articles were also categorized in terms of the informatics concepts that were applied in the research study. The concepts were data collection, data storage, data analytics, data transmission, data security, information sharing and knowledge management, and interoperability. Lastly article types were charted with the following 5 groups: qualitative, quantitative, mixed-studies, op-ed/commentaries, and literature reviews. It is important to note that op-eds/commentaries were included to adequately reflect the experts' opinions on the make-up of the field. However, it is important to note that due to the subjective nature of op-eds/commentaries, they are substantially different from original methodological articles, such as qualitative, quantitative or mixed design studies.

Following charting the articles, the total numbers of articles in each category and the intersection between categories were tallied; In addition, research gaps were identified and discussed.

Since this was a literature review study, no research ethics approval was required.

\section{Results}

The MeSH term search resulted in 1072 publications on MEDLINE/PubMed on January $3^{\text {rd }}$, 2017. A total of 515 articles met the inclusion criteria and were included in the scoping review. Figure 1 illustrates the procedure of the study selection.

Figure 2, and Tables 1, 2, 3, and 4 represent the distributions of the articles in terms of year of publication, PH domain, article type, ICT, and informatics concept, respectively. Figure 2 indicates that there has been a consistent decline in the number of articles tagged with "public health informatics" MeSH term since 2004. It is important to note that MeSH terms are occasionally added to articles several years after their publication; therefore, the figure might underestimate the extent of MeSH term usage in the recent years. The details of each $\mathrm{PH}$ domain in Table 1 are described in the ensuing sections. Table 2 indicates that about $49 \%$ of the reviewed articles are op-ed/commentaries. This large portion of op-eds/commentaries has two implications: 1) the rest of the results of this study should be interpreted with caution since opeds/commentaries do not directly represent research activity; and 2) nevertheless, they reflect the general interest level of the research community in PHI, which is important to know.

Note that there was a substantial overlap between categories; therefore, the sum of the numbers in each figure/table may be larger than the total number of reviewed articles. Table 3 illustrates the high usage of electronic registries (68.5\% of the articles) and websites $(47.6 \%)$ in the reviewed articles. 
Figures 3, 4, and 5 show the percentage distribution of the articles with respect to the article type, ICT, and informatics concept respectively in each PH domain.. According to Figure 3, oped/commentaries constitute the majority of article types in all $\mathrm{PH}$ domains except injury surveillance, which overlaps with quantitative studies the most. Figure 4, once again emphasizes the importance of websites and electronic registries in all PH domains. Moreover, high usage of GIS in environmental health could be noted in Figure 4. Lastly, Figure 5 illustrates how data security and knowledge management were not applied as much as other informatics concepts in the reviewed articles.

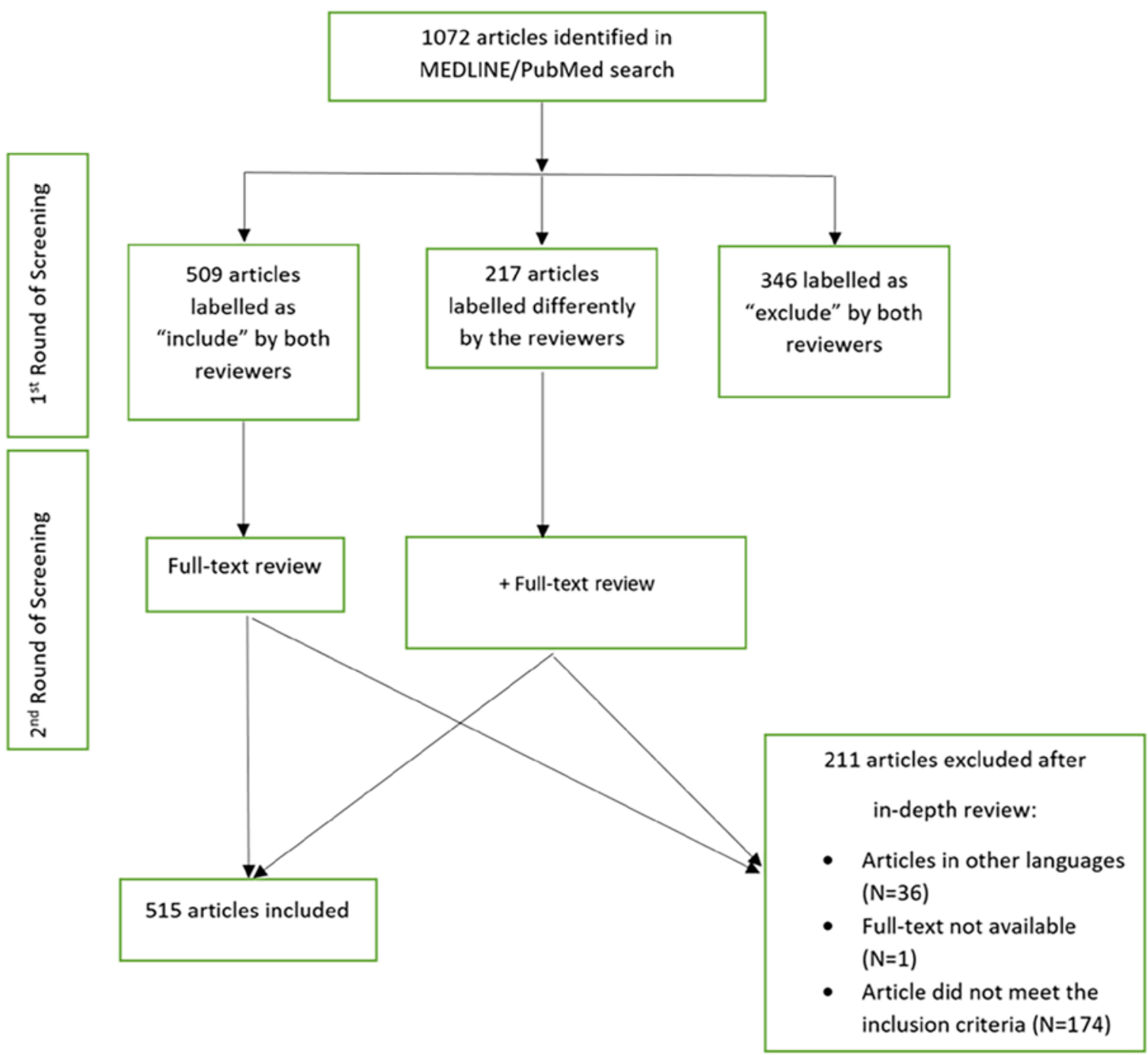

Figure 1: Flow diagram for article selection 


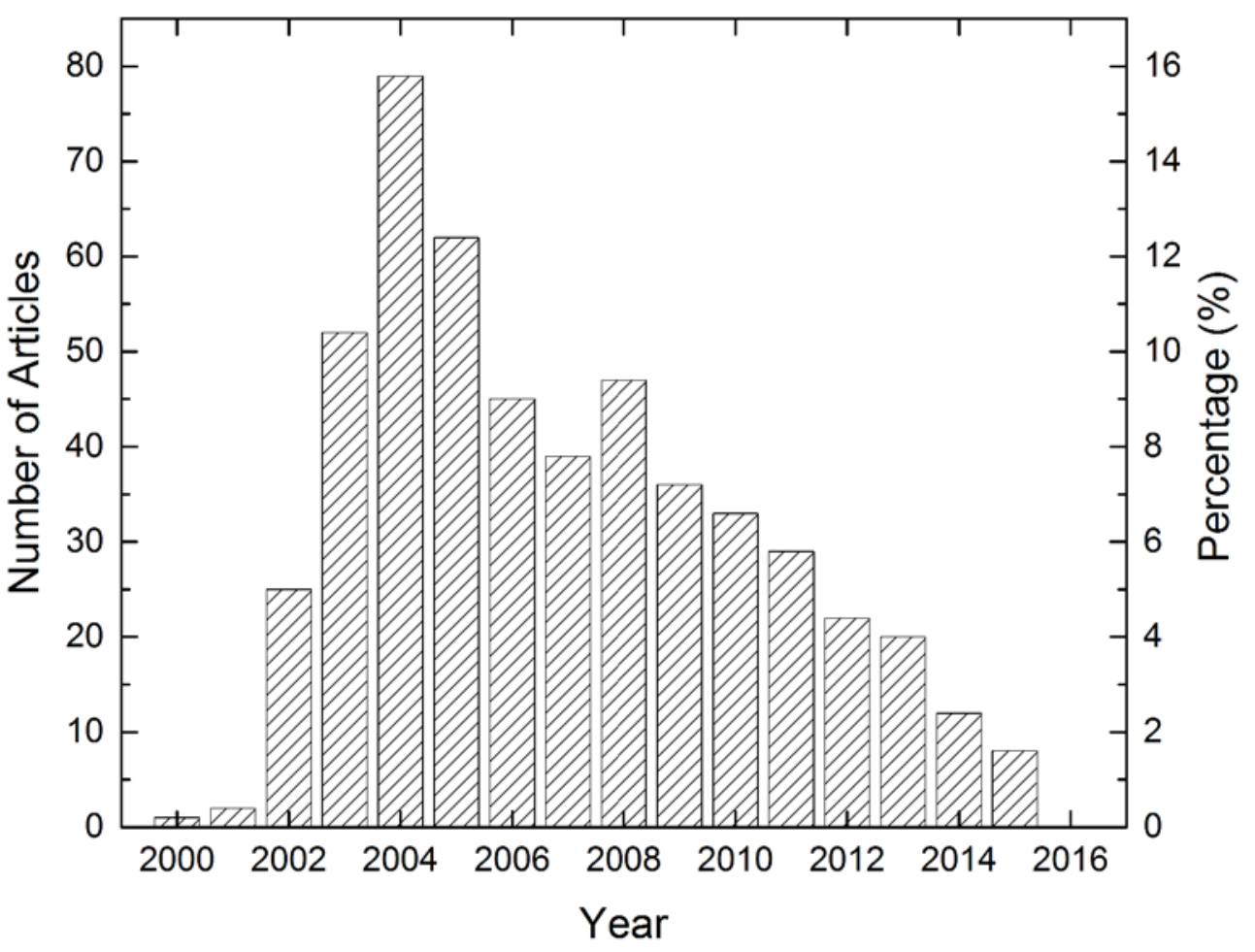

Figure 2: Article distribution in terms of year of publication

Table 1: Distribution of articles across PH domains

\begin{tabular}{|l|r|r|}
\hline \multicolumn{1}{|c|}{ PH Domain } & \multicolumn{1}{|c|}{$\begin{array}{c}\text { Number of } \\
\text { Articles }\end{array}$} & $\begin{array}{c}\text { Percentage } \\
\text { of Total } \\
\text { Number of } \\
\text { Articles (\%) }\end{array}$ \\
\hline Communicable Diseases & 232 & 45.0 \\
\hline Non-Communicable Diseases & 49 & 9.5 \\
\hline Emergency Response & 10 & 1.9 \\
\hline Injury Surveillance & 13 & 2.5 \\
\hline Natural Disaster Management & 9 & 1.7 \\
\hline Environmental Health & 17 & 3.3 \\
\hline Public Health Awareness & 85 & 38.9 \\
\hline Public Health Policy and & 201 & \\
Research & & \\
\hline
\end{tabular}


Table 2: Distribution of articles across article types

\begin{tabular}{|l|r|r|}
\hline \multicolumn{1}{|c|}{ Article Type } & $\begin{array}{c}\text { Number of } \\
\text { Articles }\end{array}$ & $\begin{array}{c}\text { Percentage } \\
\text { of Total } \\
\text { Number of } \\
\text { Articles (\%) }\end{array}$ \\
\hline Quantitative Studies & 109 & 21.2 \\
\hline Qualitative Studies & 131 & 25.4 \\
\hline Mixed Studies & 12 & 2.3 \\
\hline Op-Eds/Commentaries & 252 & 48.9 \\
\hline Review Studies & 11 & 2.1 \\
\hline
\end{tabular}

Table 3: Distribution of articles across information and communication technologies

\begin{tabular}{|l|r|r|}
\hline \multicolumn{1}{|c|}{ ICT Discussed } & $\begin{array}{c}\text { Number of } \\
\text { Articles }\end{array}$ & $\begin{array}{c}\text { Percentage } \\
\text { of Total } \\
\text { Number of } \\
\text { Articles (\%) } \\
\text { (\%) }\end{array}$ \\
\hline Detection Prediction Algorithm & 118 & 22.9 \\
\hline Electronic Registries & 353 & 68.5 \\
\hline Email & 44 & 8.5 \\
\hline GIS & 83 & 16.1 \\
\hline Mass Media & 13 & 2.5 \\
\hline Mobile Phones & 19 & 3.7 \\
\hline NLP & 21 & 4.0 \\
\hline Analytical software & 67 & 13.0 \\
\hline Landline Telephone & 45 & 8.7 \\
\hline Websites & 245 & 47.6 \\
\hline
\end{tabular}

Table 4: Distribution of articles across informatics concepts

\begin{tabular}{|l|r|r|}
\hline \multicolumn{1}{|c|}{ Informatics Concept } & $\begin{array}{c}\text { Number of } \\
\text { Articles }\end{array}$ & $\begin{array}{c}\text { Percentage of } \\
\text { Total Number } \\
\text { of Articles (\%) } \\
\text { (\%) }\end{array}$ \\
\hline Knowledge Management & 16 & 3.1 \\
\hline Data Collection & 229 & 44.4 \\
\hline
\end{tabular}




\begin{tabular}{|l|r|r|}
\hline Data Analytics & 80 & 15.5 \\
\hline Data Retrieval & 194 & 38.0 \\
\hline Data Security & 15 & 2.9 \\
\hline Data Storage & 71 & 13.8 \\
\hline Data Transmission & 279 & \\
\hline Interoperability & 132 & \\
\hline Information Sharing & 171 & 33.2 \\
\hline
\end{tabular}

Emergency Response

Natural Disaster Management

Environmental Health

Injury Surveillance

Public Health Awareness

Non-Communicable Diseases

Public Health Policy and Research

Communicable Diseases

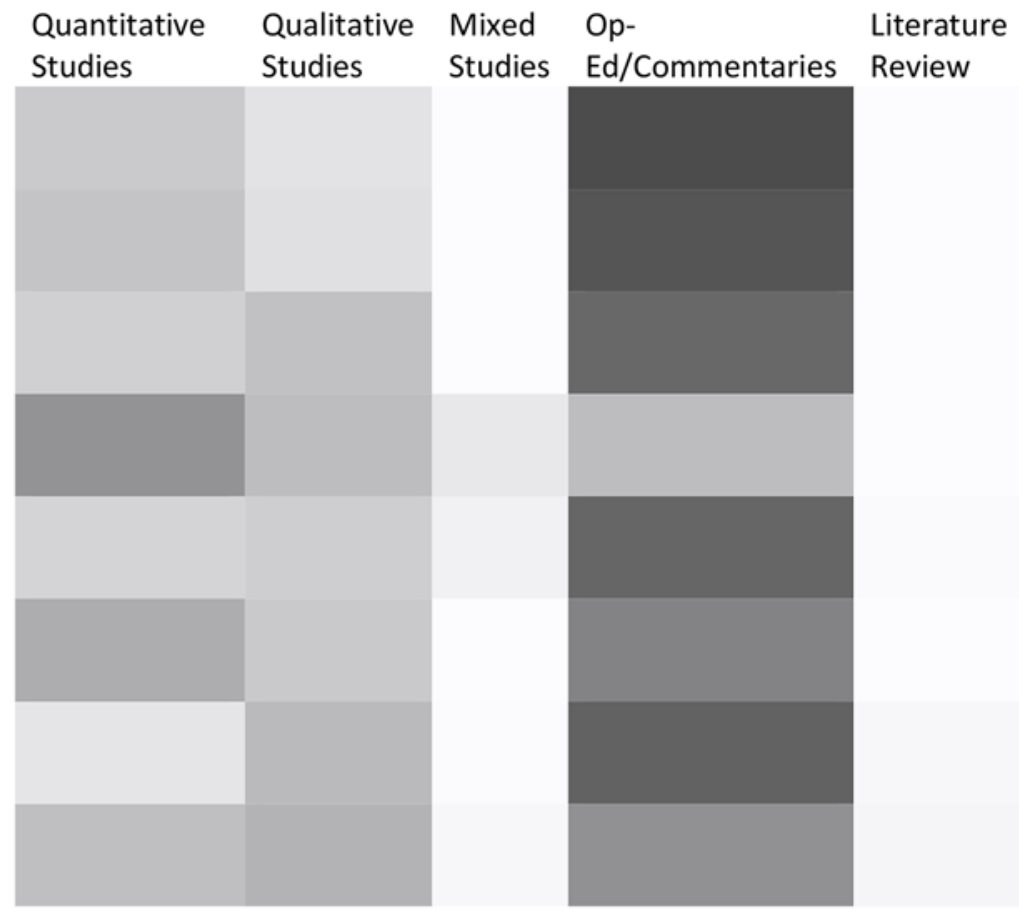

$100 \%$

$0 \%$

Note: The percentage compares the number of articles in each article type to the total number of articles in the corresponding $\mathrm{PH}$ domain

Figure 3: Distribution of articles in the intersection between each article type and PH domain 




Note: The percentage compares the number of articles in each IT to the total number of articles in the corresponding PH domain

Figure 4: Distribution of articles in the intersection between each ICT and PH domain 


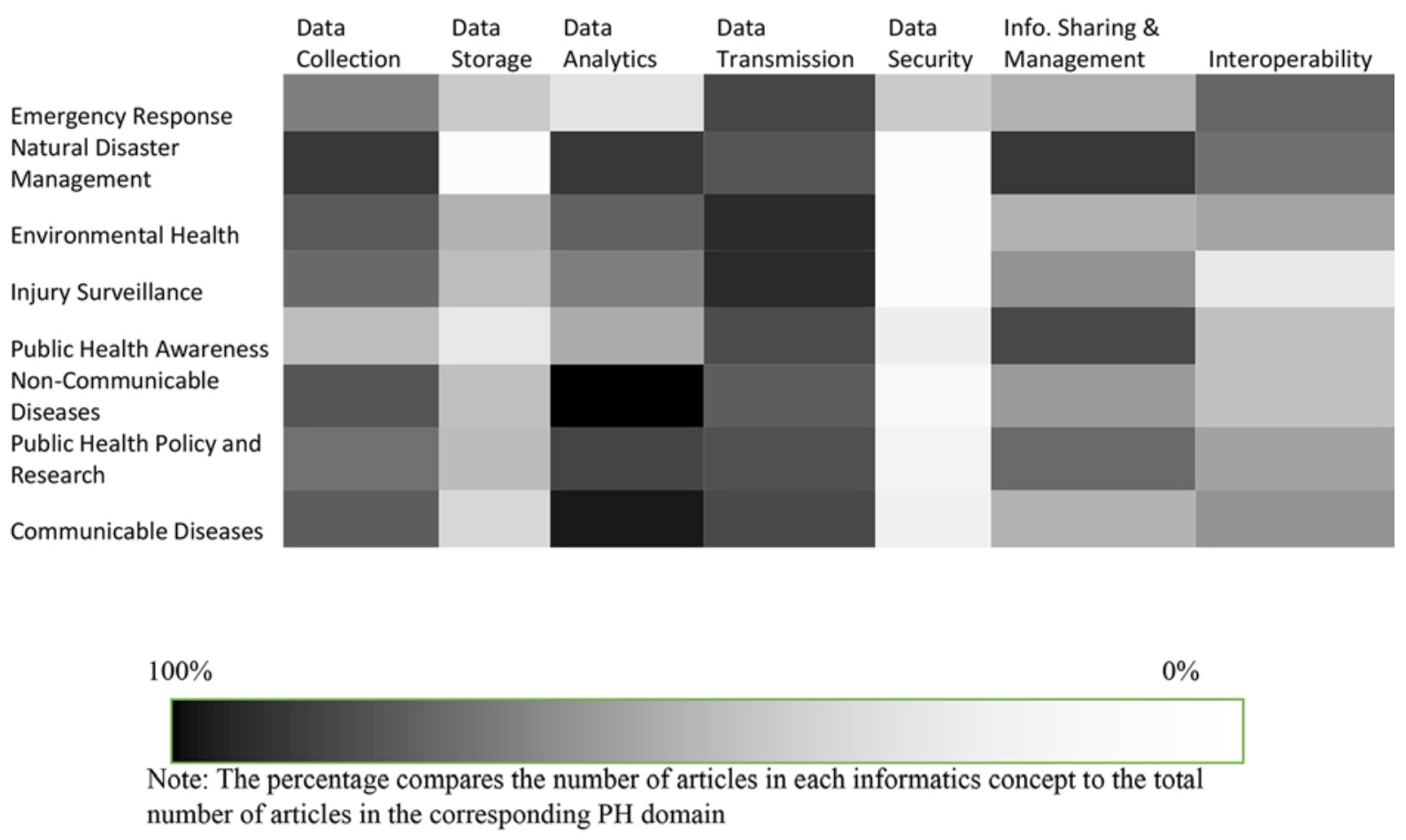

Figure 5: Distribution of articles in the intersection between each informatics concept and $\mathrm{PH}$

The full reference list of all included articles and their categories are provided in the Supplementary Material.

\section{Communicable Diseases}

As the largest public health domain, with 232 articles, this category is divided into two subcategories: infectious disease monitoring and bio-terrorism surveillance.

Infectious disease monitoring constituted 202 papers. Communication between clinical settings and $\mathrm{PH}$ units is the major theme in this sub-category. Communication can be improved through enhancement of reporting systems, web-based disease monitoring, and implementation of technologies, such as GIS and NLP. While syndromic surveillance using electronic repositories is suggested most frequently as a monitoring index, food-source monitoring, defense medical surveillance system (DMSS), and web-based data query systems are other mechanisms that could be utilized for communicable disease monitoring. Lack of Internet-based open source query systems and enhancement of data quality in this area are described as the main limitations.

About $40 \%(\mathrm{~N}=94)$ of disease surveillance articles are focused on detecting outbreaks in a timely manner. Interpretation of syndromic data with statistical techniques and DP algorithms 
has been the dominant approach to detect such outbreaks. However, inconsistent results exist regarding the validity of syndromic surveillance as a detection tool. Data from emergency departments (ED) is used as the main data source for outbreak detection; in addition, use of mobile phones, over-the-counter drug sales, and laboratory-based results are among other methods that have been used.

About 20\% ( $\mathrm{N}=45)$ of the articles focused on bio-terrorism surveillance; the main difference between these studies and the rest of this $\mathrm{PH}$ domain is the military/political focus. 88\% of bioterrorism surveillance papers were published before 2010. Electronic data reporting is discussed in $45 \%(\mathrm{~N}=21)$ of the articles. While interoperability has been illustrated as a major issue in most of these reporting systems, standardization of information infrastructure through ICD-9 and ICD10 is described to be an effective solution to integrate bio-terrorism surveillance with health care systems. Although estimates indicate that only about $25 \%$ of the affected individuals attend emergency units, the data derived from these departments have been the main source for the surveillance. Furthermore, DP algorithm has been the main technique to integrate bio-terrorism data (discussed in about $30 \%$ of the articles).

\section{Non-Communicable Disease Monitoring}

This PH domain focuses on monitoring chronic diseases and non-infectious congenital complications, such as some developmental disabilities. From the 49 articles included in this category, $47 \%(\mathrm{~N}=23)$ highlight a certain aspect of disease reporting and chronic disease surveillance. $78 \%(\mathrm{~N}=18)$ of these studies receive their data from clinical or school settings. Mother-child health has been another popular topic in this field. There are 2 articles that evaluate maternal and child health monitoring programs. These programs utilize various information technologies to enhance monitoring, such as implementing landline telephone-based surveillance for timely call response and using electronic registries to track mother and child health. Two other studies discuss congenital anomaly surveillance systems [14,15]. Low data quality is highlighted as a challenge and standardization of birth registries is proposed as a potential solution. Six studies discuss the application of GIS in chronic disease monitoring; the importance of mapping chronic diseases in terms of policy making was also discussed in all of these articles. Application of mobile phones, media, and National Health Interview Survey (NHIS) in noncommunicable disease monitoring is identified as new mechanisms to implement adequate chronic disease reporting and monitoring.

\section{Emergency Response}

The 10 articles in this category aim to detect health emergencies in order to elicit an effective response in a timely manner. Health monitoring through dispatch calls is discussed in three articles, where varying conclusions are made regarding the effectiveness of such health monitoring mechanisms. Web-based emergency services are mentioned as a tool to evaluate the available healthcare services for patients, such as hospital beds vacancy. Lastly, web-based monitoring is described as an emerging tool for health emergency response and monitoring. However, despite its recent public proliferation, no evaluations are made on the efficiency and accuracy of social media as an emergency response tool. 


\section{Environmental Health}

About half of the 17 articles in this public health domain $(\mathrm{N}=7)$ apply GIS to create a geo-spatial monitoring system. Examples of such systems include SOLAP, Internet-based GIS, and Early Warning System. Web-based databases, real-time electronic monitoring devices, and wireless communication are the main technologies that are integrated with GIS. Generally, the majority of the studies in this domain focus on the development of systems that could utilize the detection of environmental contaminations and organize the environmental data in an interoperable manner. Current environmental health programs are also assessed in three quantitative studies in which state-level public health and environmental health specialists were surveyed. It was indicated that there is a need to enhance the State-Federal interactions in the U.S. and that more environmental health data exchange is required. Moreover, the validity of ED-based data as a predictive measure for environmental health complications, such as heat waves, was questioned. It is suggested that further assessments are required to enable environmental health programs to rely on emergency-based data. Monitoring food-borne diseases, carbon monoxide detection, real-time surveillance, and implementation of new technologies, such as wearable devices that assess the air quality, are the upcoming topics in environmental health articles.

\section{Injury Surveillance}

There are a total of 13 articles in this domain. The studies are mostly focused on the development of trauma surveillance systems using injury registries. While the implementation of such injury surveillance systems in trauma units were highly recommended by 3 studies [16-18], another 2 questioned the feasibility of ED-based injury surveillance systems [19,20]. Additionally, a mixed study was conducted to evaluate a Chinese injury surveillance system based on the injury surveillance guideline developed by WHO and CDC-US 2001 [9]. It was shown that the accuracy of this system was about $80 \%$ [9]. 3 articles discussed occupational health hazard prevention by recommending the development of a reliable database that detects and stores occupational injuries. Programs such as the National Emergency Alerting and Response Systems (NEARS) [10] and European Union Public Health Information Network (EUPHIN) [11] have also proposed integration of different health systems such as bioterrorism, traffic incidents and emergency response to improve the effectiveness of surveillance.

\section{Natural Disaster Management}

9 articles discussed the application of health informatics in management of natural disasters. Among the 9 studies, emphasis on capacity building is the most common topic. As the Health Care Information and Management Systems Society (HIMSS) database indicates, about 2,667 (92\%) of the total of 2,877 healthcare organizations have wireless connection; however, the number of portable devices that could be used to manage and monitor natural disasters are less than 25\% [12]. Moreover, lack of coordination among stakeholders, the need for GIS application in healthcare and the significance of cross validation of morbidity and mortality data in natural disasters is discussed by other studies. While GIS and integrated data repositories could be useful in tackling natural disasters in developed countries, mobile phones are suggested as a promising tool for management of natural disasters in developing countries. Above all, lack of interoperability is portrayed as one of the most significant limitations of current natural disaster management in both developed and developing countries. 


\section{Public Health Awareness}

85 articles are included in this $\mathrm{PH}$ domain. 61\% $(\mathrm{N}=52)$ of these articles are focused on webbased information sharing. About 10 different web-based systems are demonstrated through which websites are used to spread health information in the population. The location of these developments is primarily in Europe and North America. For instance, the European Public Health Information and Knowledge system (EUPHIX) is a web-based system utilized to share public health-related information at the international, national, and regional level across the European Union (EU) [13]. This open-source system is developed to inform politicians, policy makers, academics, media, and the general public regarding the current state of public health by providing measurements for public health indicators or demonstrating the on-going public health activities across the EU [13]. In addition, 4 qualitative studies evaluated the current effectiveness of such health information systems using focus groups and semi-structured interviews with $\mathrm{PH}$ staff and policy makers. Although some of the content of these websites is thought to be helpful, the access is sub-optimal. It is suggested to implement a united web-portal that could be accessed easily by the public. Lastly, integration of mobile devices, email, and mass media are proposed as promising methods to share health information with the general public.

\section{Public Health Policy, System, and Research}

All of the 201 studies of this domain have a dominant focus on using informatics to aid public health policy making and/or mechanisms that could assist researchers in their PHI research. 49\% (N=99) of the articles discuss systematic integration of PHI as a significant sector of public health. Most common methods for such development are efficient data reporting and monitoring systems ( $\mathrm{N}=17$ articles), application of ICT in public health research $(\mathrm{N}=31)$, coherent PHI architectural development $(\mathrm{N}=8)$, evaluation of usefulness, data quality, effectiveness of electronic databases $(\mathrm{N}=11)$, and implementation of electronic registry in both public health policy and research $(\mathrm{N}=22)$.

$13 \%$ of the articles $(\mathrm{N}=26)$ illustrate potential mechanisms to enhance the implementation of PHI research in public health policy-making. Use of websites, surveying stakeholders' opinions, building an inter-disciplinary infrastructure for PHI research, and translating research to practice are among other significant mechanisms that are discussed in the reviewed articles. Health data exchange is another aspect of public health policy and research, which is emphasized in $11 \%$ $(\mathrm{N}=23)$ of the articles. 7 of the 24 studies highlight the effectiveness of real time data sharing between clinical and public health units. Moreover, 16 articles discuss potential data linkage systems that connect various jurisdictions of public health research and policy. Lastly, web-based information sharing is another area of data linkage systems that works through open source databases. Security and data quality of such systems are thought to be the main challenges to their global implementation. Other topics in this public health domain include children health information, GIS and public health research, clinical decisions support systems, and users' knowledge and practice in application of ICT in disease prevention.

\section{Discussion}

While there are 1072 articles tagged with the MeSH term "public health informatics", only about half of them had a major focus on application of ICT to public health (see Figure 1). This 
discrepancy indicates that: 1) MeSH terms may not necessarily represent the corresponding field of study, possibly due to a lack of standardization and/or guidelines for MeSH term assignment; and 2) PHI sometimes does not necessarily imply use of ICT. This finding also speaks to the interdisciplinary nature of health informatics. At least partially overlapping definitions of health informatics sub-fields complicate distinguishing various disciplines from each other.

Disease and injury surveillance, environmental monitoring, health promotion, and research/policy utilization were the main public health themes that were observed in the 515 reviewed papers. There have been numerous approaches for each of these fields and enhancements could be easily observed in many areas of PHI. More comprehensive repositories, promotion of open-source databases and real-time monitoring facilities, emphasis on capacity building, quality assurance, and application of new technologies, such as GIS and NLP, are some of such improvements. Despite these advancements, there are several gaps that need to be filled in order to take full advantage of informatics in public health. The following points illustrate some of the observations made throughout this scoping review:

1. About $70 \%(\mathrm{~N}=353)$ of the articles focused on EHR and electronic repositories, which are becoming the dominant platform for collection, storage, and analysis of health data, particularly in the U.S. These health data have substantial research and public health value and could affect the healthcare system dramatically. However, there is a need to standardize the data in such repositories. Considering the large volume of data that will be accumulated in the coming years, it will be virtually impossible to derive any valid interpretation from the repositories if the entered data does not follow a standardized structure. As a result, standardization of health data, such as using consistent ICD codes, could enhance the usability of repositories in PHI.

2. Only $12 \%(\mathrm{~N}=5)$ of the bio-terrorism articles have been published since 2010. This dramatic decline in bio-terrorism studies could indicate a potential area of research in the functionality of current bio-terrorism surveillance systems that have not been assessed in the past 7 years. Examples of such systems include BioStorm \& EARS, NEARS, BioSense, NRDM, and National Bio-terrorism Syndromic Surveillance Demonstration Program. Another possible interpretation of this trend could be a decline in tagging bio-terrorism surveillance studies with "public health informatics" MeSH term. Considering that bio-terrorism is one of the fundamental components of PHI, this decline could indicate the lack of inclusivity and consistency of MeSH term assignment. Overall more research needs to be done to investigate such decline in more depth.

3. Inconsistency in the adequacy of surveillance methodologies is highlighted many times in number of articles. For instance, various predictors are used for disease surveillance and emergency monitoring, such as syndromic surveillance, dispatch calls, OTC drug sale, and school absenteeism. However, there are inconsistent results regarding the validity of most of these indexes. More research should be conducted to further investigate the validity of such predictors. In addition, data sources seem to be sub-optimal. Although a large number of articles in communicable disease monitoring and injury surveillance used ED-based data, the validity of this data source is subpar due to the low attendance of patients to emergency departments. Therefore, more research needs to be done on either 
using a more comprehensive data source or identifying the most optimal combination of data sources. Generally, considering the importance of surveillance systems in PHI, optimizing data sources and predictor variables could be a significant contribution to the healthcare system. With more valid measurements and accurate surveillance systems, PHI could establish a strong foundation in our healthcare system and could be implemented as a tool to optimize public health policy making and practice.

4. Electronic health data exchange was emphasized several times throughout this scoping review. Data and information sharing could help public health sectors communicate with one another and strengthen public health's coherence. More commonly done through websites and Internet-based reporting, electronic health data exchange has become a promising approach to increase PHI's interoperability. However, data privacy is an issue that needs to be ensured, particularly with large-scale health data. Moreover, the quality of the data collected at clinical facilities is another major concern for stakeholders. In most clinical units, clinicians are in charge of entering and editing the health data into electronic repositories. As a result, the secondary use of the data solely depends on the clinicians' understanding of research and policy-making. Consequently, lack of health informatics training in the clinical sector, could lead to low quality data entry for PHI research purposes.

Overall, PHI is a multi-disciplinary field with promising ideas and complex challenges. There are a large number of stakeholders involved in public health, such as policy makers, clinicians, non-government organizations, and the public itself. This diversity in stakeholders makes decision-making and communication between the parties difficult. However, through the advantages of informatics, decision-making, disease surveillance, communication, and public health research could be enhanced as long as the stakeholders overcome related challenges, such as funding, interoperability and quality of data sources.

\section{Limitations}

Relying solely on MeSH terms rather than searching keywords was a main limitation of this study. For instance, searching the keyword "public health informatics" in PubMed results in several articles that this study did not cover, such as "health informatics and the H1N1 pandemic [5], "Design principles in the development of (public) health informatics infrastructures" [21], "An informatics framework to support surveillance system interoperability in Minnesota" [22], "The centers of excellence in public health informatics: improving public health through innovation, collaboration, dissemination, and translation" [23], and "Beyond information access: support for complex cognitive activities in public health informatics tools" [24]. Hence, for a more comprehensive review study on the entire PHI field, it would be necessary to expand search terms and inclusion criteria.

Another limitation of this scoping review was that only one database was searched. More relevant articles are likely also available in other databases. For example, the Online Journal of Public Health Informatics (OJPHI) has been publishing 3 issues a year since 2009. The OJPHI also publishes the Proceedings of the International Society for Disease Surveillance (ISDS). While most of the papers published in OJPHI directly address the application of ICTs to public 
health practice and research, these papers are indexed in PubMed and are excluded from this study. Future researchers must expand the search term to cover public health informatics papers published in other health services journals. Lastly, sometimes MeSH terms are assigned months to years after the publication of a research article. As a result, there is a chance that several studies were missed due to the fact that they were not assigned with a MeSH term yet.

\section{Conclusions}

In the past 20 years, PHI has evolved to encompass numerous sub-categories, each of which focuses on a particular aspect of public health. ICT is increasingly becoming the main focus of PHI research and practice. Furthermore, this diverse range of subcategories, including both the technological and public health aspects of PHI, could be utilized comprehensively to assist policy makers and strengthen public health measures. Overcoming the current challenges could facilitate this comprehensive integration of PHI into the healthcare system. The current challenges include enhancement of interoperability, maintaining high data quality, appropriate data sources, accessible health information, and communication between stakeholders. Further research in these areas could be an adequate starting point to tackle these challenges.

\section{Acknowledgements}

Aein Zarrin and Arjun Kumar Bhattarai both contributed equally to this research study. The authors would like to thank the University of Waterloo for general research support. The authors also acknowledge the guidance of Jackie Stapleton and Rebecca Hutchinson, librarians at the University of Waterloo, provided regarding the literature search.

\section{Financial Disclosure}

This work was supported by a Discovery Grant (RGPIN-2014-04743) from the Natural Sciences and Engineering Research Council of Canada (NSERC). AKB was funded by the School of Public Health and Health Systems, University of Waterloo. AZ was funded by Undergraduate Student Research Award from NSERC as well as Undergraduate Research Internship program at the University of Waterloo.

\section{Competing Interests}

The authors have no competing interests.

\section{References}

1. Yasnoff WA, Overhage JM, Humphreys BL, LaVenture M. 2001. A national agenda for public health informatics: summarized recommendations from the 2001 AMIA Spring Congress. J Am Med Inform Assoc. 8(6), 535-45. PubMed https://doi.org/10.1136/jamia.2001.0080535

2. Baker EL, Fond M, Hale P, Cook J. 2016. What Is 'Informatics'? J Public Health Manag Pract. 22(4), 420-23. PubMed https://doi.org/10.1097/PHH.0000000000000415 
3. Last JM. 2001. A dictionary of epidemiology. 4th Ed. Oxford University Press; 2001.

4. Friede A, Blum HL, Mcdonald M. 1995. Public-health informatics-how information-age technology can strengthen public-health. Annu Rev Public Health. 16, 239-52. PubMed https://doi.org/10.1146/annurev.pu.16.050195.001323

5. Lombardo J. 2012. Public health informatics and the H1N1 pandemic. Online J Public Health Inform. 1(1), Epub

6. U.S. National Library of Medicine. Introduction to MeSH. Available at: https://www.nlm.nih.gov/mesh/introduction.html

7. Dixon BE, Pina J, Kharrazi H, Gharghabi F, Richards J. 2015. What's Past is Prologue: A Scoping Review of Recent Public Health and Global Health Informatics Literature. Online $J$ Public Health Inform. 7(2). Epub. 06, 2015.

8. Ritchie J, Spencer L. Qualitative data analysis for applied policy research. London, U.K.: Routledge, 1994.

9. Liu X, Li L, Cui H, Liu X, Jackson VW. 2009. Evaluation of an emergency departmentbased injury surveillance project in China using WHO guidelines. Inj Prev. 15(2), 105-10. PubMed https://doi.org/10.1136/ip.2008.019877

10. Woodhall J. Homeland security, car crashes and traffic incident data: Killing three birds with one IT architecture. 2005. Journal of Safety Research. 36(5). 461-463. PubMed https://doi.org/10.1016/j.jsr.2005.10.001

11. European Union Public Health Information Network (The Euphin). 2003. Eur J Public Health. 13(3), 114-15. PubMed

12. Harrison JP, Harrison RA, Smith M. 2008. Role of information technology in disaster medical response. Health Care Manag. 27(4), 307-13. PubMed

13. Achterberg PW, Kramers PGN, van Oers HM. 2008. European community health monitoring: the EUPHIX-model. Scand J Public Health. 36(7), 676-84. PubMed

14. Lowry RB. ... Congenitals anomalies surveillance in Canada. Can J Public Health. 99(6), 483-85. PubMed

15. Suresh S, Thangavel G, Sujatha J, Indrani S. 2005. Natl Med J India. 18(5), 259-62. PubMed

16. Brown JA. 2008. An internet database for the classification and dissemination of information about hazardous chemicals and occupational diseases. Am J Ind Med. 51(6), 428-35. PubMed https://doi.org/10.1002/ajim.20578

17. Cinnamon J \& Schuurman N. 2010. Injury surveillance in low-resource settings using Geospatial and Social Web technologies. Int J Health Georg. 9(25). 
18. Downing A, Wilson R. 2005. Regional surveillance of accident and emergency departmnt attendances: experiences from the West midlands. J Public Health. 27(1), 82-84. PubMed https://doi.org/10.1093/pubmed/fdh190

19. Newcombe RG, Lyons RA, Jones SJ, Patterson J. Home injuries and built form methodological issues and developments in database linkage. BMC Health Serv. Res.5(12), Epub 042004.

20. Petridou E, Dessypris N, Frangakis CE, Belechri M, Mavrou A, et al. 2004. Estimating the population burden of injuries: a comparison of household surveys and emergency department surveillance. Epidemiology. 15(4), 428-32. PubMed https://doi.org/10.1097/01.ede.0000129519.76945.0d

21. Neame R. 2012. Design principles in the development of (public) health informatics infrastructures. Online J Public Health Inform. 4(10). Epub. 05, 2017.

22. Patnoe ST, LeVenture M, Johnson RE, Fritz J, Frohnert B, et al. 2016. An informatics framework to support surveillance system interoperability in Minesota. Online J Public Health Inform. 9(1).

23. Husting LE, Gadsden-Knowles K. 2011. The centers of excellence in public health informatics: improving public health through innovation, collaboration, dissemination, and translation. Online J Public Health Inform, 3(3). Epub. 12, 2011.

24. Sedig K, Parsons P, Dittmer M, Ola O. 2012. Beyond information access: support for complex cognitive activities in public health informatics tools. Online J Public Health Inform, 4(3). Epub. 12, 2012. 